\title{
Role of 1,4-dimethylpiperazine in radical cyclizations
}

\author{
Tsuyoshi Taniguchi, Ryota Kawajiri, and Hiroyuki Ishibashi* \\ Division of Pharmaceutical Sciences, Graduate School of Natural Science and Technology, \\ Kanazawa University, Kanazawa 920-1192, Japan \\ E-mail: isibasi@p.kanazawa-u.ac.jp
}

\begin{abstract}
Radical cyclization of $o$-ethenyltrichloroacetanilides in boiling 1,4-dimethylpiperazine was examined with a comparison of the mode of radical cyclizations under a $\mathrm{Bu}_{3} \mathrm{SnH}$-mediated condition. It was found that an attack of a hydrogen atom on the cyclized radical intermediates occurred more rapidly in 1,4-dimethylpiperazine than under the $\mathrm{Bu}_{3} \mathrm{SnH}$-mediated condition. This phenomenon was also observed in similar reactions of $\mathrm{N}$-ethenyltrichloroacetamide.
\end{abstract}

Keywords: Neophyl rearrangement, radical cyclization, single electron transfer, trichloroacetamide

\section{Introduction}

A previous study in our laboratory revealed that treatment of $N$-allylic trichloroacetamides $\mathbf{1}$ in boiling 1,4-dimethylpiperazine gave the corresponding 5-membered lactams 2 in good yields (Scheme 1). ${ }^{1}$ Formation of 2 might be a result of radical cyclization that proceeded via a single electron transfer reaction from the nitrogen atom of 1,4-dimethylpiperazine to the precursor 1. This reaction will provide an unprecedented method for radical cyclization without using a combination of a radical initiator such as azobis(isobutyronitrile) (AIBN) and a hydrogen atom donor such as tributyltin hydride $\left(\mathrm{Bu}_{3} \mathrm{SnH}\right){ }^{2-8}$

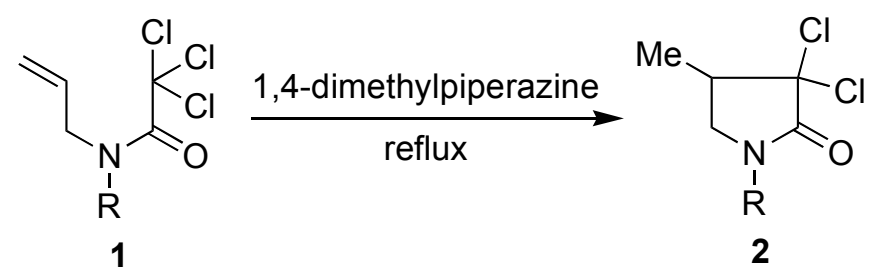

\section{Scheme 1}

We have now examined the reaction of $o$-ethenyltrichloroacetanilides in boiling 
1,4-dimethylpiperazine with a comparison of the mode of radical cyclizations under a $\mathrm{Bu}_{3} \mathrm{SnH}$-mediated condition, and found that an attack of a hydrogen atom on the cyclized radical intermediates occurred more rapidly in 1,4-dimethylpiperazine than under the $\mathrm{Bu}_{3} \mathrm{SnH}$-mediated condition. This phenomenon was also observed in similar reactions of $\mathrm{N}$-ethenyltrichloroacetamide. The results of our works in this area are presented in this paper.

\section{Results and Discussion}

$o$-Ethenyltrichloroacetanilide 3 was prepared by acylation of $o$-ethenyl- $N$-methylaniline with trichloroacetyl chloride. When compound 3 was heated in boiling 1,4-dimethylpiperazine for 20 min, cyclized product 4 was obtained in $76 \%$ yield (Scheme 2). On the other hand, treatment of 3 with $\mathrm{Bu}_{3} \mathrm{SnH}$ in the presence of AIBN (using the slow addition technique) in boiling chlorobenzene gave two compounds 5 and $\mathbf{6}$ in 32\% and 25\% yields, respectively.<smiles>C=Cc1ccccc1N(C)C(=O)C(Cl)(Cl)Cl</smiles>

3

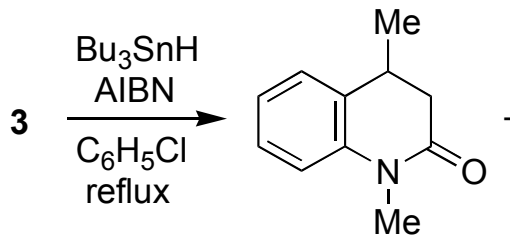

5: $32 \%$

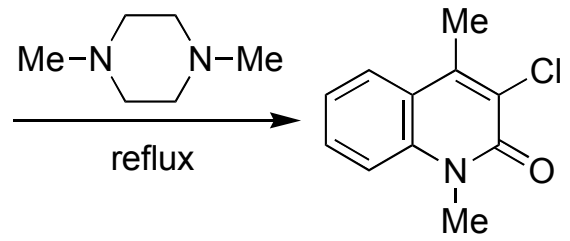

4: $76 \%$

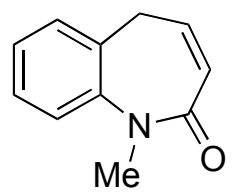

6: $25 \%$

\section{Scheme 2}

Formation of 4 from 3 can be explained as follows (Scheme 3). A single electron transfer reaction from a nitrogen atom of 1,4-dimethylpiperazine (1,4-DMP) to the starting compound 3 gives radical anion $\mathbf{A}$ together with radical cation $\mathbf{B}$. Removal of the chloride anion of $\mathbf{A}$ affords radical $\mathbf{C}$, which then undergoes cyclization in a 6-exo-trig manner to give cyclized radical $\mathbf{D}$. An attack of radical $\mathbf{D}$ on the hydrogen atom of radical cation $\mathbf{B}$ or 1,4-dimethylpiperazine (1,4-DMP) itself followed by elimination of hydrogen chloride gives the observed product 4 . 


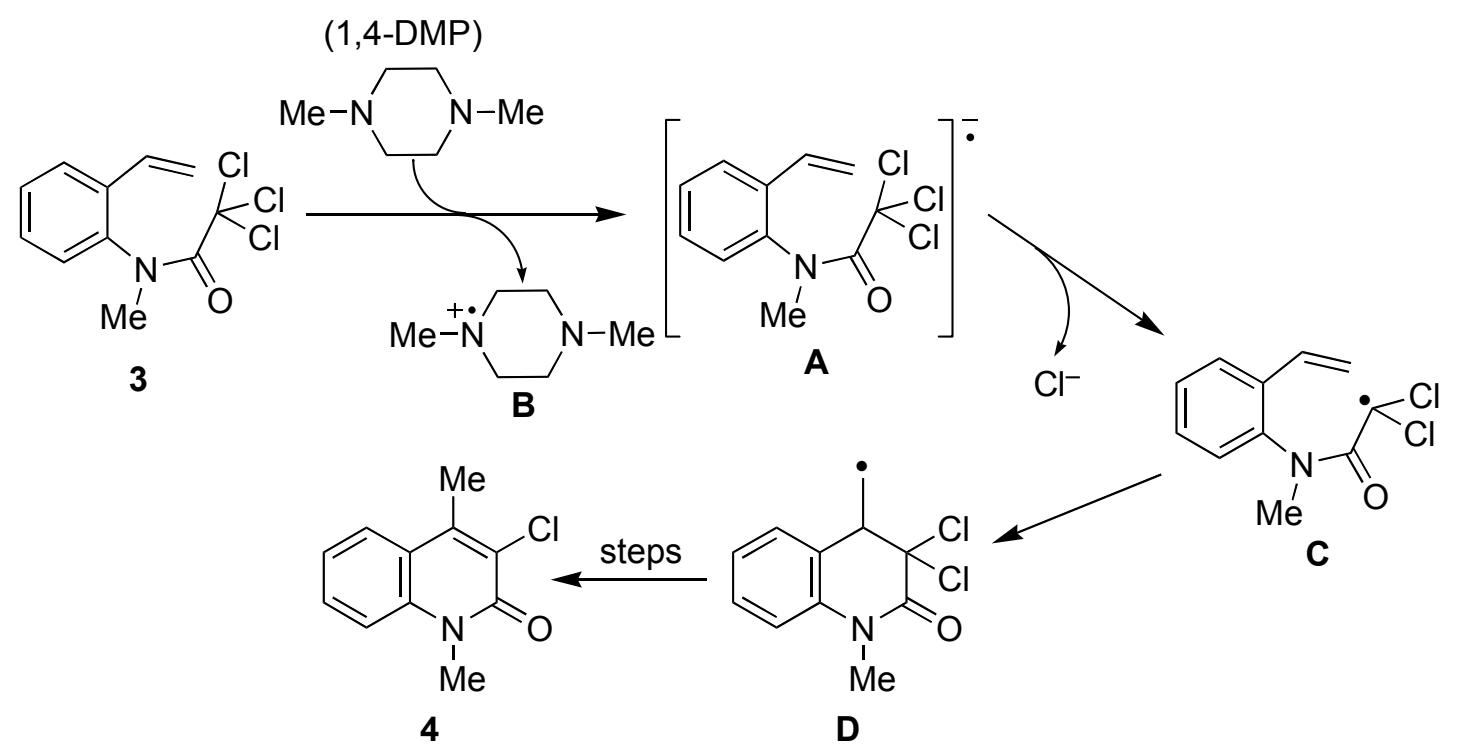

\section{Scheme 3}

Formation of $\mathbf{5}$ from $\mathbf{3}$ may proceed via an attack of $\mathrm{Bu}_{3} \mathrm{SnH}$ on the radical center of $\mathbf{D}$ and successive reduction of the two chlorine atoms by $\mathrm{Bu}_{3} \mathrm{SnH}$. Formation of 7 -membered unsaturated lactam 6 was presumed to be not a result of a direct 7-endo cyclization of the radical formed from $\mathbf{3}$ but a result of a neophyl rearrangement of intermediate $\mathbf{D}$ to give $\mathbf{F}$ through the intermediacy of radical $\mathbf{E}$ (Scheme 4).<smiles>CC1c2ccccc2N(C)C(=O)C1(Cl)Cl</smiles>

\section{Scheme 4}

When the concentration of the hydrogen atom source is high in the radical reaction media, an initially cyclized radical intermediate (such as $\mathbf{D}$ ) is rapidly reduced by an attack of a hydrogen atom, whereas when the concentration of the hydrogen atom source is low, the cyclized radical can undergo another reaction such as neophyl rearrangement before attack by a hydrogen atom. Therefore, no formation of the neophyl rearrangement product in boiling 1,4-dimethylpiperazine might indicate that the concentration of the hydrogen atom source was high, and formation of neophyl rearrangement product $\mathbf{6}$ from $\mathbf{3}$ showed that the concentration of the hydrogen atom source under the $\mathrm{Bu}_{3} \mathrm{SnH}$-mediated condition was low.

Treatment of compound 7 in boiling 1,4-dimethylpiperazine afforded 8 and 9 in 65\% and 14\% 
yields, respectively, whereas reaction of 7 under the $\mathrm{AIBN}-\mathrm{Bu}_{3} \mathrm{SnH}$ condition gave the products $\mathbf{1 0}$ and $\mathbf{1 1}$ in $40 \%$ and $29 \%$ yields, respectively (Scheme 5).<smiles>CCc1c(Cl)c(=O)n(C)c2ccccc12</smiles>

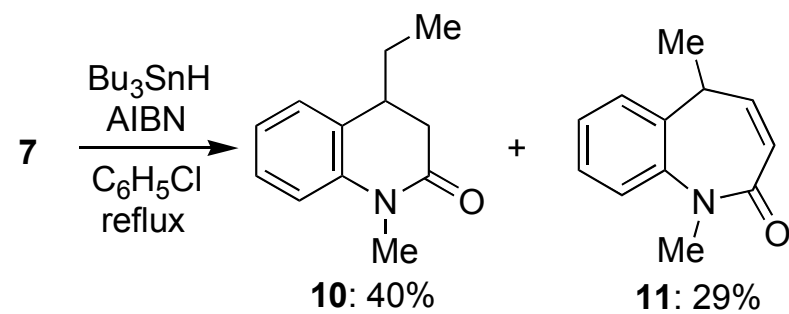

\section{Scheme 5}

It is assumed that compounds $\mathbf{8}$ and $\mathbf{1 0}$ are 6-exo cyclization products and that compounds $\mathbf{9}$ and $\mathbf{1 1}$ are neophyl rearrangement products. The product ratio of 6-exo cyclization product 8 and a neophyl rearrangement product 9 in boiling 1,4-dimethylpiperazine was 4.6:1 and that of 10:11 under the $\mathrm{Bu}_{3} \mathrm{SnH}$-mediated condition was 1.4:1. These results also indicated that the concentration of the hydrogen atom source was higher in boiling 1,4-dimethylpiperpazine than under the $\mathrm{Bu}_{3} \mathrm{SnH}$-mediated condition.

The cyclization of 12, which was prepared by $N$-methylation of $o$-(1-methylethenyl)trichloroacetanilide, in boiling 1,4-dimethylpiperazine gave 6-exo cyclization products $\mathbf{1 3}$ and $\mathbf{1 4}$ and the neophyl rearrangement product $\mathbf{1 5}$ in 33\%, 11\% and 36\% yields, respectively, and the reaction of $\mathbf{1 2}$ with $\mathrm{AIBN}-\mathrm{Bu}_{3} \mathrm{SnH}$ gave the neophyl rearrangement product 15 and 16 and 7-endo cyclization product 17 in 10\%,36\% and 5\% yields, respectively (Scheme 6). No formation of a 6-exo cyclization product under the $\mathrm{Bu}_{3} \mathrm{SnH}$-mediated conditions also strongly indicated that the concentration of the hydrogen atom source under the $\mathrm{Bu}_{3} \mathrm{SnH}$-mediated condition was low. 
<smiles>C=C(C)c1ccccc1N(C)C(=O)C(Cl)(Cl)Cl</smiles>

12<smiles>CN1CCN(C)C(Cc2ccc3c(c2)C(C)(C)C(Cl)(Cl)C(=O)N3C)C1</smiles>

13: $33 \%$<smiles>CN1C(=O)C(Cl)C(C)(C)c2ccccc21</smiles>

14: $11 \%$<smiles>CC1=C(Cl)C(=O)N(C)c2ccccc2C1</smiles>

15: $36 \%$

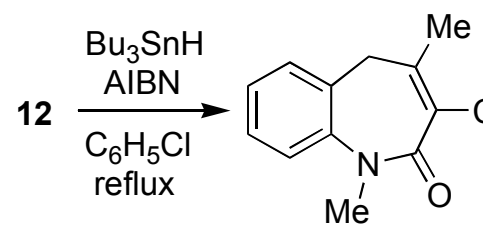

15: $10 \%$<smiles></smiles>

16: $36 \%$<smiles>CC1CCC(=O)N(C)c2ccccc21</smiles>

17: $5 \%$

\section{Scheme 6}

It is recognized that 5-endo-trig cyclization of 4-pentenyl radicals is a disfavored process due to the stereoelectronic disadvantage in the attack of the radical center on the alkenic bond. ${ }^{9} \mathrm{We}$ have found, however, that 5-endo-trig radical cyclizations occurred smoothly in some $\mathrm{N}$-vinylic $\alpha$-haloacetamides. ${ }^{10}$ We turned our attention to enamide 18, which was prepared by trichloroacetylation of imine from 2,2-bis(phenylthio)acetaldehyde and benzylamine.

We could not isolate any cyclized product by heating 18 in boiling 1,4-dimethylpiperazine but obtained mono-dechlorinated compound 19 in $26 \%$ yield (Scheme 7 ). On the other hand, compound 20 was isolated in 18\% yield together with several unidentified products by treatment of 18 with $\mathrm{AIBN}$ and $\mathrm{Bu}_{3} \mathrm{SnH}$ in boiling chlorobenzene.

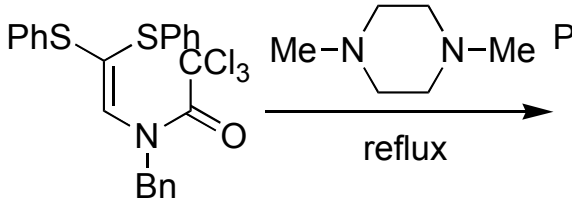

18<smiles>O=C([SnH2])N(C=C([SnH3])[SbH])Cc1ccccc1</smiles>

19: $26 \%$

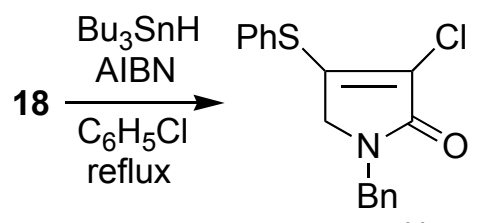

20: $18 \%$

\section{Scheme 7}

Formation of $\mathbf{2 0}$ could be explained by 5-endo-trig cyclization of radical $\mathbf{G}$ followed by chemical transformations of the resultant radical intermediate $\mathbf{H}$ (Scheme 8). 


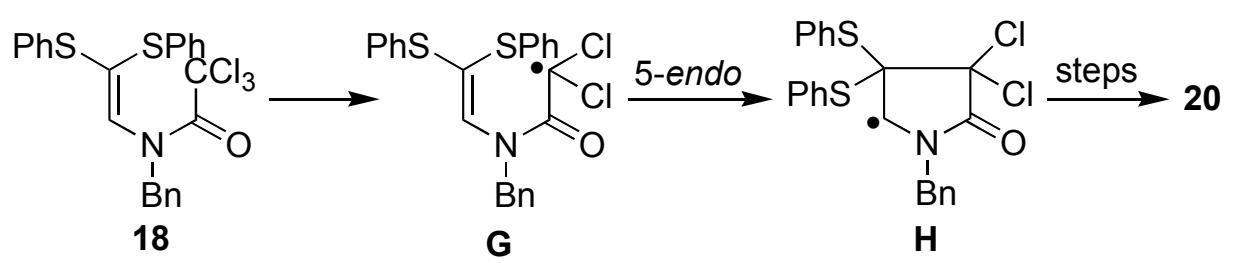

\section{Scheme 8}

When the concentration of the hydrogen atom source is high, a 4-pentenyl radical intermediate such as $\mathbf{G}$ may be reduced immediately by a hydrogen atom. Therefore, it is assumed that heating 18 in boiling 1,4-dimethylpiparazine, in which the concentration of the hydrogen atom source is high, gives the reduction product, and cyclized products are obtained under the $\mathrm{Bu}_{3} \mathrm{SnH}$-mediated condition, in which the concentration of the hydrogen atom source is low.

In conclusion, radical cyclizations of a range of 2-ethenyltrichloroacetanilides in boiling 1,4-dimethylpiperazine and under $\mathrm{Bu}_{3} \mathrm{SnH}$-mediated conditions have revealed that the condition using 1,4-dimethylpiperazine works more effectively as a hydrogen atom donor than does the condition using $\mathrm{Bu}_{3} \mathrm{SnH}$.

\section{Experimental Section}

2'-Ethenyl- $N$-methyltrichloroacetanilide (3). To a solution of 2-ethenyl- $N$-methylaniline ${ }^{11}$ (344 $\mathrm{mg}, 2.58 \mathrm{mmol})$ in $\mathrm{CH}_{2} \mathrm{Cl}_{2}(10 \mathrm{~mL})$ were added trichloroacetyl chloride $(0.35 \mathrm{~mL} .3 .10 \mathrm{mmol})$ and triethylamine $(0.54 \mathrm{~mL}, 3.87 \mathrm{mmol})$ at $0{ }^{\circ} \mathrm{C}$ and the mixture was stirred at room temperature for 10 min. The reaction mixture was diluted with water and extracted $\mathrm{CH}_{2} \mathrm{Cl}_{2}$, and the extract was dried $\left(\mathrm{MgSO}_{4}\right)$ and concentrated. The residue was chromatographed on silica gel (hexane/AcOEt, 20:1) to give $3(691 \mathrm{mg}, 96 \%)$ as an oil, whose ${ }^{1} \mathrm{H}$ and ${ }^{13} \mathrm{C}$ NMR spectra showed it to be a mixture of rotamers; IR $\left(\mathrm{CHCl}_{3}\right) \mathrm{cm}^{-1}: 1680 ;{ }^{1} \mathrm{H} \mathrm{NMR}\left(270 \mathrm{MHz}, \mathrm{CDCl}_{3}\right) \delta: 3.32(3 \mathrm{H} \mathrm{x} \mathrm{3/5,} \mathrm{br} \mathrm{s),} 3.62(3 \mathrm{H} \mathrm{x}$ 2/5, br s), 5.41 (1H, d, $J=11.0 \mathrm{~Hz}), 5.80(1 \mathrm{H}, \mathrm{dd}, J=17.5,1.0 \mathrm{~Hz}), 6.77(1 \mathrm{H}, \mathrm{m}), 7.26-7.40$ (3H, m), $7.63(1 \mathrm{H}, \mathrm{d}, J=7.6 \mathrm{~Hz}) ;{ }^{13} \mathrm{C} \mathrm{NMR}\left(125 \mathrm{MHz}, \mathrm{CDCl}_{3}\right) \delta: 42.2,80.4,117.3,126.2,128.3,129.1$, 129.4, 131.0, 131.9, 135.4, 140.1; HRMS Calcd for $\mathrm{C}_{11} \mathrm{H}_{10} \mathrm{Cl}_{3} \mathrm{NO}$ : 276.9828, found: 276.9826 .

3-Chloro-1,4-dimethylquinolin-2(1H)-one (4). A solution of compound 3 (163 mg, $0.59 \mathrm{mmol}$ ) in 1,4-dimethylpiperazine $(60 \mathrm{~mL})$ was heated at reflux for $20 \mathrm{~min}$. The reaction mixture was diluted with water and extracted with $\mathrm{CH}_{2} \mathrm{Cl}_{2}$. The organic layer was dried $\left(\mathrm{MgSO}_{4}\right)$ and concentrated, and the residue was chromatographed on silica gel (hexane/AcOEt, 10:1) to give 4 (94 mg, 76\%) as colorless crystals; mp 186-186.5 ${ }^{\circ} \mathrm{C}$ (from hexane). IR $\left(\mathrm{CHCl}_{3}\right) \mathrm{cm}^{-1}: 1640 .{ }^{1} \mathrm{H}$ NMR $\left(270 \mathrm{MHz}, \mathrm{CDCl}_{3}\right) \delta: 2.65(3 \mathrm{H}, \mathrm{s}), 3.80(3 \mathrm{H}, \mathrm{s}), 7.30(2 \mathrm{H}, \mathrm{ddd}, J=8.1,7.1,1.2 \mathrm{~Hz}), 7.39(1 \mathrm{H}$, $\mathrm{dd}, J=8.4,0.8 \mathrm{~Hz}), 7.59(1 \mathrm{H}$, ddd, $J=8.7,7.2,1.5 \mathrm{~Hz}), 7.77(1 \mathrm{H}, \mathrm{dd}, J=8.1,1.5 \mathrm{~Hz}) ;{ }^{13} \mathrm{C}$ NMR $(125$ $\left.\mathrm{MHz}, \mathrm{CDCl}_{3}\right) \delta: 16.4,30.7,114.5,120.6,122.6,125.4,126.3,130.4,137.9,142.4$, 158.0. Anal. Calcd for $\mathrm{C}_{11} \mathrm{H}_{10}$ CINO: C, 63.62; H, 4.85; N, 6.75. Found: C, 63.70; H, 4.84; N, 6.79. 
3,4-Dihydro-1,4-dimethylquinolin-2(1H)-one

(5)

and 1,5-dihydro-1-methyl-2H-1benzazepin-2-one (6). A solution of $\mathrm{AIBN}$ (16 mg, $0.1 \mathrm{mmol})$ and $\mathrm{Bu}_{3} \mathrm{SnH}(218 \mathrm{mg}, 0.75 \mathrm{mmol})$ in chlorobenzene $(25 \mathrm{~mL}$ ) was added dropwise using a syringe pump to a solution of $\mathbf{3}$ (139 $\mathrm{mg}$, $0.5 \mathrm{mmol})$ in chlorobenzene $(25 \mathrm{~mL})$ at reflux during $1.5 \mathrm{~h}$. After consumption of the starting material, to the reaction mixture was added a solution of AIBN (16 mg, $0.1 \mathrm{mmol})$ and $\mathrm{Bu}_{3} \mathrm{SnH}$ $(218 \mathrm{mg}, 0.75 \mathrm{mmol})$ in chlorobenzene $(2 \mathrm{~mL})$ and the mixture was heated at reflux for $30 \mathrm{~min}$. The solvent was removed and the residue was chromatographed on silica gel containing KF (10\%) (hexane/AcOEt, 4:1). The first eluate gave $5^{12}(28 \mathrm{mg}, 32 \%)$ as an oil; IR $\left(\mathrm{CHCl}_{3}\right) \mathrm{cm}^{-1}: 1655 ;{ }^{1} \mathrm{H}$ $\operatorname{NMR}\left(500 \mathrm{MHz}, \mathrm{CDCl}_{3}\right) \delta: 1.28(3 \mathrm{H}, \mathrm{d}, J=7.1 \mathrm{~Hz}), 2.46(1 \mathrm{H}, \mathrm{dd}, J=15,9,7.6 \mathrm{~Hz}), 2.73(1 \mathrm{H}, \mathrm{dd}$, $J=15.8,5.5 \mathrm{~Hz}), 3.02-3.10(1 \mathrm{H}, \mathrm{m}), 3.37(3 \mathrm{H}, \mathrm{s}), 6.99-7.07(2 \mathrm{H}, \mathrm{m}), 7.19-7.30(2 \mathrm{H}, \mathrm{m}) ;{ }^{13} \mathrm{C} \mathrm{NMR}$ $\left(125 \mathrm{MHz}, \mathrm{CDCl}_{3}\right) \delta: 19.2,29.4,30.3,39.1,114.7,123.0,126.2,127.4,131.0,139.8,169.8$; HRMS Calcd for $\mathrm{C}_{11} \mathrm{H}_{13} \mathrm{NO}$ : 175.0997, found: 175.0996. The second eluate gave 6 (22 mg, 25\%) as an oil; IR $\left(\mathrm{CHCl}_{3}\right) \mathrm{cm}^{-1}: 1665 ;{ }^{1} \mathrm{H}-\mathrm{NMR}\left(500 \mathrm{MHz}, \mathrm{CDCl}_{3}\right) \delta: 3.29(2 \mathrm{H}, \mathrm{br}), 3.51(3 \mathrm{H}, \mathrm{s}), 5.91$ $(1 \mathrm{H}, \mathrm{d}, J=10.7 \mathrm{~Hz}), 6.60(1 \mathrm{H}, \mathrm{dt}, J=10.7,2.9 \mathrm{~Hz}), 7.10-7.15(2 \mathrm{H}, \mathrm{m}), 7.22-7.29(2 \mathrm{H}, \mathrm{m}) ;{ }^{13} \mathrm{C}$ NMR $\left(125 \mathrm{MHz}, \mathrm{CDCl}_{3}\right) \delta: 32,1,37.0,122.1 .125 .3,125.4,127.2,127.7,136.6,141.3,141.8,167.2$; HRMS Calcd for $\mathrm{C}_{11} \mathrm{H}_{11} \mathrm{NO}$ : 173.0841, found: 173.0839 .

N-Methyl-2'-(1-propenyl)trichloroacetanilide (7). According to a procedure similar to that described above for 3, $N$-methyl-2-(1-propenyl)aniline ${ }^{13}(1.79 \mathrm{~g}, 12.09 \mathrm{mmol})$ was acetylated with trichloroacetyl chloride $(1.6 \mathrm{~mL} .14 .51 \mathrm{mmol})$ to give $7(1.78 \mathrm{~g}, 81 \%)$ as colorless crystals, whose ${ }^{1} \mathrm{H}$ and ${ }^{13} \mathrm{C}$ NMR spectra showed it to be a mixture of rotamers; mp $88-89{ }^{\circ} \mathrm{C}$ (from hexane); ${ }^{1} \mathrm{H}$ $\operatorname{NMR}\left(500 \mathrm{MHz}, \mathrm{CDCl}_{3}\right) \delta: 1.91(3 \mathrm{H}, \mathrm{d}, J=5.6 \mathrm{~Hz}), 3.31$ (3H x 9/14, br s), 3.59 (3H x 5/14, br s), 6.22-6.44 (2H, m), 7.24-7.36 (3H, m), $7.54(1 \mathrm{H}, \mathrm{d}, J=7.6 \mathrm{~Hz}) ;{ }^{13} \mathrm{C} \mathrm{NMR}\left(125 \mathrm{MHz}, \mathrm{CDCl}_{3}\right) \delta: 18.9$, $42.1,126.0,126.1,126.7,127.1,128.4,129.0,129.5,135.8,139.4,161.0$. Anal. Calcd for $\mathrm{C}_{12} \mathrm{H}_{12} \mathrm{Cl}_{3} \mathrm{NO}$ : C, 49.26; H, 4.13; N, 4.79. Found: C, 49.25; H, 4.13; N, 4.80.

\section{3-Chloro-4-ethyl-1-methylquinolin-2(1H)-one (8) and 3-chloro-1,5-dihydro-1,5-} dimethyl-2H-1-benzazepin-2-one (9). A solution of compound 7 (146 mg. $0.5 \mathrm{mmol}$ ) in 1,4-dimethylpiperazine $(2 \mathrm{~mL})$ was heated at reflux for $20 \mathrm{~min}$. A work-up similar to that described above for $\mathbf{4}$ and the crude materials were purified by chromatography on silica gel (hexane/AcOEt, 10:1). The first eluate gave 9 (16 mg, 14\%) as an oil; IR $\left(\mathrm{CHCl}_{3}\right) \mathrm{cm}^{-1}: 1645 ;{ }^{1} \mathrm{H}$ $\operatorname{NMR}\left(500 \mathrm{MHz}, \mathrm{CDCl}_{3}\right) \delta: 1.53(3 \mathrm{H}, \mathrm{d}, J=7.1 \mathrm{~Hz}), 3.55(3 \mathrm{H}, \mathrm{s}), 3.57-3.58(1 \mathrm{H}, \mathrm{m}), 6.43(1 \mathrm{H}, \mathrm{d}$, $J=5.9 \mathrm{~Hz}), 7.23-7.50(4 \mathrm{H}, \mathrm{m}) ;{ }^{13} \mathrm{C} \mathrm{NMR}\left(125 \mathrm{MHz}, \mathrm{CDCl}_{3}\right) \delta:$ 15.7, 33.2. 38.0, 122.6, 122.9, 125.9, 127.0, 128.8, 140.3, 141.2, 144.6, 163.4; HRMS Calcd for $\mathrm{C}_{12} \mathrm{H}_{12} \mathrm{ClNO}$ : 221.0607, found: 221.0610. The second eluate gave $8\left(72 \mathrm{mg}, 65 \%\right.$ ) as colorless crystals; mp $91.5-92{ }^{\circ} \mathrm{C}$ (from hexane); IR $\left(\mathrm{CHCl}_{3}\right) \mathrm{cm}^{-1}: 1640 .{ }^{1} \mathrm{H} \mathrm{NMR}\left(500 \mathrm{MHz}, \mathrm{CDCl}_{3}\right) \delta: 1.28(3 \mathrm{H}, \mathrm{t}, J=7.6 \mathrm{~Hz}), 3.10(2 \mathrm{H}$, q, $J=7.6 \mathrm{~Hz}), 3.79(3 \mathrm{H}, \mathrm{s}), 7.27-7.32(1 \mathrm{H}, \mathrm{m}), 7.39(1 \mathrm{H}, \mathrm{d}, J=8.5), 7.57-7.60(1 \mathrm{H}, \mathrm{m}), 7.77(1 \mathrm{H}, \mathrm{d}$, $J=8.3 \mathrm{~Hz}) .{ }^{13} \mathrm{C} \mathrm{NMR}\left(125 \mathrm{MHz}, \mathrm{CDCl}_{3}\right) \delta: 12.5,23.3,30.7,114.7,119.4,122.6,125.1,125.4$, 130.3, 138.3, 147.8, 158.1. Anal. Calcd for $\mathrm{C}_{12} \mathrm{H}_{12} \mathrm{ClNO}$ : C, 65.02; H, 5.46; N, 6.32. Found: C, $64.88 ; \mathrm{H}, 5.46 ; \mathrm{N}, 6.53$.

4-Ethyl-3,4-dihydro-1-methylquinolin-2(1H)-one

(10) and 1,5-dihydro-1,5-dimethyl2H-1-benzazepin-2-one (11). Using a procedure similar to that described above for the 
preparation of 5 and 6, compound 7 (146 mg, $0.5 \mathrm{mmol}$ ) was treated twice with AIBN (16 mg, 0.1 $\mathrm{mmol})$ and $\mathrm{Bu}_{3} \mathrm{SnH}(218 \mathrm{mg}, 0.75 \mathrm{mmol})$. After a usual work-up, the residue was chromatographed on silica gel (hexane/AcOEt, 5:1). The first eluate gave $\mathbf{1 0}^{11}(38 \mathrm{mg}$. 40\%) as an oil; IR $\left(\mathrm{CHCl}_{3}\right) \mathrm{cm}^{-1}: 1660 ;{ }^{1} \mathrm{H}$ NMR $\left(500 \mathrm{MHz}, \mathrm{CDCl}_{3}\right) \delta: 0.93(3 \mathrm{H}, \mathrm{t}, J=7.6 \mathrm{~Hz}), 1.52-1.64(2 \mathrm{H}$, m), $2.61(1 \mathrm{H}, \mathrm{dd}, J=11.6,6.1 \mathrm{~Hz}), 2.73-2.77(2 \mathrm{H}, \mathrm{m}), 3.35(3 \mathrm{H}, \mathrm{s}), 6.99-7.04(2 \mathrm{H}, \mathrm{m}), 7.14-7.16$ $(1 \mathrm{H}, \mathrm{m}), 7.24-7.29(1 \mathrm{H}, \mathrm{m}) ;{ }^{13} \mathrm{C} \mathrm{NMR}\left(125 \mathrm{MHz}, \mathrm{CDCl}_{3}\right) \delta: 11.4,26.4,29.2,36.6,37.6,114.8$, 122.6, 127.3, 127.7, 129.7, 139.7, 169.7. The second eluate gave $\mathbf{1 1}(27 \mathrm{mg}, 29 \%)$ as an oil; IR $\left(\mathrm{CHCl}_{3}\right) \mathrm{cm}^{-1}: 1655 ;{ }^{1} \mathrm{H}$ NMR $\left(500 \mathrm{MHz}, \mathrm{CDCl}_{3}\right) \delta: 1.53(3 \mathrm{H}, \mathrm{d}, J=6.8 \mathrm{~Hz}), 3.51(3 \mathrm{H}, \mathrm{s}), 3.62(1 \mathrm{H}$, br s), $5.82(1 \mathrm{H}, \mathrm{d}, J=10.5 \mathrm{~Hz}), 6.28(1 \mathrm{H}, \mathrm{br}), 7,17-7.28(4 \mathrm{H}, \mathrm{m}) ;{ }^{13} \mathrm{C} \mathrm{NMR}\left(125 \mathrm{MHz}, \mathrm{CDCl}_{3}\right) \delta$ : 16.0, 29.7, 37.1, 122.3, 123.4, 125.4, 126.8, 127.1, 140.6, 141.7, 148.5, 167.3; HRMS Calcd for $\mathrm{C}_{12} \mathrm{H}_{13} \mathrm{NO}$ : 187.0997, found: 187.0997.

N-Methyl-2'-(1-methylethenyl)trichloroacetanilide (12). According to a procedure similar to that described above for 3, 2'-(1-methylethenyl)trichloroacetanilide (2.20 g, quant.) was prepared from 2-(1-methylethenyl)aniline $(1.0 \mathrm{~mL}, 7.34 \mathrm{mmol})$ and trichloroacetyl chloride $(1.0 \mathrm{~mL}, 8.81$ $\mathrm{mmol})$. To a suspension of sodium hydride $(289 \mathrm{mg}$. $7.22 \mathrm{mmol})$ in a combined solvent of THF and $\mathrm{DMF}=1: 1(2 \mathrm{~mL})$ was added a solution of 2'-(1-methylethenyl)trichloroacetanilide (1.34 g, 4.81.mmol) in THF $(4 \mathrm{~mL})$ at $0{ }^{\circ} \mathrm{C}$ and the mixture was stirred at the same temperature for $10 \mathrm{~min}$. Methyl iodide $(0.45 \mathrm{~mL}, 7.22 \mathrm{mmol})$ was added to the mixture and the mixture was stirred at room temperature for $30 \mathrm{~min}$. The reaction mixture was diluted with water and extracted with AcOEt. The organic layer was washed with brine, dried $\left(\mathrm{MgSO}_{4}\right)$, and concentrated. The residue was chromatographed on silica gel (hexane/AcOEt, 30:1) to give $\mathbf{1 2}(1.18 \mathrm{~g}, 84 \%)$ as colorless crystals, whose ${ }^{1} \mathrm{H}$ and ${ }^{13} \mathrm{C}$ NMR spectra showed it to be a mixture of rotamers; mp $35-35.5{ }^{\circ} \mathrm{C}$ (from hexane); IR $\left(\mathrm{CHCl}_{3}\right) \mathrm{cm}^{-1}: 1685 ;{ }^{1} \mathrm{H}$ NMR $\left(270 \mathrm{MHz}, \mathrm{CDCl}_{3}\right) \delta: 2.08(3 \mathrm{H}, \mathrm{s}), 3.31(3 \mathrm{H} \mathrm{x} \mathrm{1/5,} \mathrm{br} \mathrm{s})$, $3.62\left(3 \mathrm{H} \mathrm{x} \mathrm{4/5,} \mathrm{s),} 4.98(1 \mathrm{H}, \mathrm{br} \mathrm{s}), 5.21(1 \mathrm{H}, \mathrm{br} \mathrm{s}), 7.19-7.35(4 \mathrm{H}, \mathrm{m}) ;{ }^{13} \mathrm{C}-\mathrm{NMR}\left(125 \mathrm{MHz}, \mathrm{CDCl}_{3}\right)\right.$ $\delta: 23.4,41.7,115.9,127.3,127.9,128.2,128.4,129.7,140.8,141.3,142.1,160.0$. Anal. Calcd for $\mathrm{C}_{12} \mathrm{H}_{12} \mathrm{Cl}_{3} \mathrm{NO}$ : C, 49.26; H, 4.13; N, 4.79. Found: C, 49.15; H, 4.11; N, 4.72.

3,3-Dichloro-3,4-dihydro-1,4,4-trimethylquinolin-2(1H)-one (13), 3-chloro-3,4-dihydro1,4,4-trimethylquinolin-2(1H)-one (14) and 3-chloro-1,5-dihydro-1,4-dimethyl-2H-1benzazepin-2-one (15). A solution of compound 12 (146 mg. $0.5 \mathrm{mmol})$ in 1,4-dimethylpiperazine $(2 \mathrm{~mL})$ was heated at reflux for $20 \mathrm{~min}$. After a work-up similar to that described above for 4, the residue was chromatographed on silica gel (hexane/AcOEt, 10:1). The first eluate gave 13 (43 mg, 33\%) as colorless crystals; mp 96-97 ${ }^{\circ} \mathrm{C}$ (from hexane); IR $\left(\mathrm{CHCl}_{3}\right.$ ) $\mathrm{cm}^{-1}: 1690 ;{ }^{1} \mathrm{H}$ NMR (500 MHz, $\left.\mathrm{CDCl}_{3}\right) \delta: 1.30(3 \mathrm{H}, \mathrm{s}), 1.80(3 \mathrm{H}, \mathrm{s}), 3.52(3 \mathrm{H}, \mathrm{s}), 7.07(1 \mathrm{H}, \mathrm{d}$, $J=8.3 \mathrm{~Hz}), 7.14-7.17(1 \mathrm{H}, \mathrm{m}), 7.32-7.37(2 \mathrm{H}, \mathrm{m}) ;{ }^{13} \mathrm{C} \mathrm{NMR}\left(125 \mathrm{MHz}, \mathrm{CDCl}_{3}\right) \delta: 20.8,25.1,31.5$, 46.8, 92.6, 115.2, 124.3, 125.1, 128.2, 131.7, 137.3, 161.9. Anal. Calcd for $\mathrm{C}_{12} \mathrm{H}_{13} \mathrm{Cl}_{2} \mathrm{NO}$ : C, 55.83; H, 5.08; N, 5.43. Found: C, 55.66; H, 5.07; N, 5.36. The second eluate gave 14 (12 mg, $11 \%$ ) as colorless crystals; $\mathrm{mp} 91-92{ }^{\circ} \mathrm{C}$ (from hexane); IR $\left(\mathrm{CHCl}_{3}\right) \mathrm{cm}^{-1}: 1680 ;{ }^{1} \mathrm{H} \mathrm{NMR}(500$ $\left.\mathrm{MHz} \mathrm{CDCl}_{3}\right) \delta: 1.31(3 \mathrm{H}, \mathrm{s}), 1.48(3 \mathrm{H}, \mathrm{s}), 3.44(3 \mathrm{H}, \mathrm{s}), 4.25(1 \mathrm{H}, \mathrm{s}), 7.06-7.34(4 \mathrm{H}, \mathrm{m}) ;{ }^{13} \mathrm{C} \mathrm{NMR}$ $\left(125 \mathrm{MHz}, \mathrm{CDCl}_{3}\right) \delta: 23.1,26.1,30.0,38.5,64.7,115.1,123.9,125.2,127.9,131.2,138.0,165.3$. Anal. Calcd for $\mathrm{C}_{12} \mathrm{H}_{14} \mathrm{ClNO}$ : C, 64.43; H, 6.31; N, 6.26. Found: C, 64.08; H, 6.30; N, 6.30. The 
third eluate gave 15 (40 mg, 36\%) as an oil; IR $\left(\mathrm{CHCl}_{3}\right) \mathrm{cm}^{-1}: 1645 ;{ }^{1} \mathrm{H} \mathrm{NMR}\left(500 \mathrm{MHz}, \mathrm{CDCl}_{3}\right) \delta$ : $2.11(3 \mathrm{H}, \mathrm{s}), 2.99(1 \mathrm{H}, \mathrm{d}, J=13.4 \mathrm{~Hz}), 3.55(3 \mathrm{H}, \mathrm{s}), 3.64(1 \mathrm{H}, \mathrm{d}, J=13.4 \mathrm{~Hz}), 7.13-7.31(4 \mathrm{H}, \mathrm{m}) ;{ }^{13} \mathrm{C}$ NMR (125 MHz, $\left.\mathrm{CDCl}_{3}\right) \delta: 21.8,37.6,37.9,121.0,122.2,125.7,127.0,127.6,135.6,141.2,146.5$, 164.1; HRMS Calcd for $\mathrm{C}_{12} \mathrm{H}_{12} \mathrm{ClNO}$ : 221.0607, found: 221.0606 .

1,5-Dihydro-1,4-dimethyl-2H-1-benzazepin-2-one (16) and 1,3,4,5-tetrahydro-1,5dimethyl-2H-1-benzazepin-2-one (17). Using a procedure similar to that described above for the preparation of 5 and 6, compound $12(146 \mathrm{mg}, 0.5 \mathrm{mmol})$ was treated twice with AIBN (16 mg, 0.1 $\mathrm{mmol})$ and $\mathrm{Bu}_{3} \mathrm{SnH}(218 \mathrm{mg}, 0.75 \mathrm{mmol})$. After a usual work-up, the residue was chromatographed on silica gel (hexane/AcOEt, 3:1). The first eluate gave $17^{14}(5 \mathrm{mg}, 5 \%)$ as an oil; IR $\left(\mathrm{CHCl}_{3}\right) \mathrm{cm}^{-1}: 1650 ;{ }^{1} \mathrm{H}$ NMR $\left(500 \mathrm{MHz}, \mathrm{CDCl}_{3}\right) \delta: 1.35(3 \mathrm{H}, \mathrm{d}, J=6.8 \mathrm{~Hz}), 1.56-1.63(1 \mathrm{H}$, m), 2.20-2.30 (2H, m), 2.37-2.45 (1H, m), 2.99-3.07 (1H, m), $3.34(3 \mathrm{H}, \mathrm{s}), 7.16-7.31(4 \mathrm{H}, \mathrm{m}) ;{ }^{13} \mathrm{C}$ NMR $\left(125 \mathrm{MHz}, \mathrm{CDCl}_{3}\right) \delta: 17.8,32.6,33.5,35.1,37.5,122.3,125.2,126.2,127.0,138.6,143.5$, 173.4. The second eluate gave $\mathbf{1 5}(11 \mathrm{mg}, 10 \%)$ as an oil. The third eluate gave $\mathbf{1 6}^{11}(34 \mathrm{mg}, 36 \%)$ as colorless crystals; mp 95.5-96.5 ${ }^{\circ} \mathrm{C}$ (from hexane), lit. ${ }^{11} 96-97{ }^{\circ} \mathrm{C}$ (from hexane); IR $\left(\mathrm{CHCl}_{3}\right.$ ) $\mathrm{cm}^{-1}: 1660 ;{ }^{1} \mathrm{H}$ NMR $\left(500 \mathrm{MHz}, \mathrm{CDCl}_{3}\right) \delta: 1.97(3 \mathrm{H}, \mathrm{s}), 3.24(2 \mathrm{H}, \mathrm{br}), 3.49(3 \mathrm{H}, \mathrm{s}), 5.69(1 \mathrm{H}, \mathrm{s})$, 7.09-7.28 (4H, m); ${ }^{13} \mathrm{C} \mathrm{NMR}\left(125 \mathrm{MHz}, \mathrm{CDCl}_{3}\right) \delta: 24.3,36.6,38.0,120.0,121.6,125.1,127.2$, $127.3,135.5,141.8,152.8,167.4$.

$\boldsymbol{N}$-Benzyl- $\boldsymbol{N}$-[2,2-bis(phenylthio)ethenyl]trichloroacetamide (18). To a solution of 2,2-bis(phenylthio)acetaldehyde ${ }^{15}(1.44 \mathrm{~g}, 5.33 \mathrm{mmol})$ in $\mathrm{Et}_{2} \mathrm{O}(30 \mathrm{~mL})$ were added benzylamine $(0.6 \mathrm{~mL}, 5.33 \mathrm{mmol})$ and $\mathrm{MgSO}_{4}(10 \mathrm{~g})$, and the mixture was stirred at room temperature for $3 \mathrm{~h}$. The reaction mixture was filtered and the filtrate was concentrated. To a solution of this residue in toluene $(50 \mathrm{~mL})$ were added trichloroacetyl chloride $(0.93 \mathrm{~mL}, 8.3 \mathrm{mmol})$ and $N, N$-diethylaniline $(1.76 \mathrm{ml}, 11.06 \mathrm{mmol})$ and the mixture was heated under reflux for $2 \mathrm{~h}$. The reaction mixture was diluted with water and extracted with AcOEt. The extract was washed with brine, dried $\left(\mathrm{MgSO}_{4}\right)$, and concentrated. The residue was chromatographed on silica gel (hexane/AcOEt, 60:1 $\rightarrow 30: 1$ ) to give $18(2.18 \mathrm{~g}, 80 \%)$ as an oil; IR $\left(\mathrm{CHCl}_{3}\right) \mathrm{cm}^{-1}: 1685 ;{ }^{1} \mathrm{H} \mathrm{NMR}\left(270 \mathrm{MHz}, \mathrm{CDCl}_{3}\right) \delta: 5.12(2 \mathrm{H}$, s), $6.87(1 \mathrm{H}, \mathrm{s}), 7.05-7.39(15 \mathrm{H}, \mathrm{m}) ;{ }^{13} \mathrm{C} \mathrm{NMR}\left(125 \mathrm{MHz}, \mathrm{CDCl}_{3}\right) \delta: 54.0,92.9,127.9,128.0$, 128.2, 128.4, 128.71, 128.73, 128.9. 129.8, 130.5, 131.8, 132.5, 133.3, 135.1, 135.9, 160.4; HRMS Calcd for $\mathrm{C}_{23} \mathrm{H}_{18} \mathrm{Cl}_{3} \mathrm{NOS}_{2}$ : 492.9896 , found: 492.9893 .

$\boldsymbol{N}$-Benzyl- $\boldsymbol{N}$-[2,2-bis(phenylthio)ethenyl]dichloroacetamide (19). A solution of compound 18 (49.5 mg, $0.1 \mathrm{mmol})$ in 1,4-dimethylpiperazine $(5 \mathrm{~mL})$ was heated at reflux for $30 \mathrm{~min}$. After a work-up similar to that described above for 4 , the residue was chromatographed on silica gel (hexane/AcOEt, 30:1 $\rightarrow 10: 1)$ to give $19(12 \mathrm{mg}, 26 \%)$ as an oil; IR $\left(\mathrm{CHCl}_{3}\right) \mathrm{cm}^{-1}: 1690 ;{ }^{1} \mathrm{H} \mathrm{NMR}$ $\left(500 \mathrm{MHz} \mathrm{CDCl}_{3}\right) \delta: 4.81(2 \mathrm{H}, \mathrm{s}), 6.24(1 \mathrm{H}, \mathrm{s}), 6.36(1 \mathrm{H}, \mathrm{br}), 7.08-7.44(15 \mathrm{H}, \mathrm{m}) ;{ }^{13} \mathrm{C}-\mathrm{NMR}$ $\left(125 \mathrm{MHz}, \mathrm{CDCl}_{3}\right) \delta: 51.1,65.1,126.7,127.9,128.4,128.7,128.9,129.1,129.7,131.6,132.3$, 133.8, 135.5, 139.6, 163.5; HRMS Calcd for $\mathrm{C}_{23} \mathrm{H}_{19} \mathrm{Cl}_{2} \mathrm{NOS}_{2}$ : 459.0285, found: 459.0280 .

1-Benzyl-3-chloro-2-oxo-4-phenylthio-3-pyrroline (20). Using a procedure similar to that described above for the preparation of 5 and 6, compound $\mathbf{1 8}$ (1.34 g, $2.71 \mathrm{mmol})$ was treated twice with $\mathrm{AIBN}(89 \mathrm{mg}, 0.54 \mathrm{mmol})$ and $\mathrm{Bu}_{3} \mathrm{SnH}(867 \mathrm{mg}, 2.98 \mathrm{mmol})$. After a usual work-up, the residue was chromatographed on silica gel (hexane/AcOEt, 10:1 $\rightarrow 5: 1 \rightarrow 3: 1$ ) to give 20 (154 
mg, 18\%) as colorless crystals; mp 38-39 ${ }^{\circ} \mathrm{C}$; IR $\left(\mathrm{CHCl}_{3}\right) \mathrm{cm}^{-1}: 1695 ;{ }^{1} \mathrm{H}$ NMR $\left(500 \mathrm{MHz}, \mathrm{CDCl}_{3}\right)$ $\delta: 3.53(2 \mathrm{H}, \mathrm{s}), 4.55(2 \mathrm{H}, \mathrm{s}), 7.14(2 \mathrm{H}, \mathrm{d}, J=6.7 \mathrm{~Hz}), 7.23-7.50(8 \mathrm{H}, \mathrm{m}) ;{ }^{13} \mathrm{C} \mathrm{NMR}(125 \mathrm{MHz}$, $\left.\mathrm{CDCl}_{3}\right) \delta: 46.9,51.6,121.1,127.3,127.68,127.75,128.7,129.8,130.0,134.7,136.4,146.7$, 165.2; HRMS Calcd for $\mathrm{C}_{17} \mathrm{H}_{14}$ CINOS: 315.0485 , found: 315.0491 .

\section{Acknowledgements}

This work was supported by a Grant-in-Aid for Scientific Research from the Ministry of Education, Culture, Sports, Science and Technology of Japan.

\section{References}

1. Ishibashi, H.; Haruki, S.; Uchiyama, M.; Tamura, O.; Matsuo, J. Tetrahedron Lett. 2006, 47, 6263.

2. Baguley, P. A.; Walton, J. C. Angew. Chem. Int. Ed. 1998, 37, 3072.

3. Studer, A.; Amrein, S. Synthesis 2002, 835.

4. Martin, C. G.; Murphy, J. A.; Smith, C. R. Tetrahedron Lett. 2000, 41, 1833.

5. Jang, D. O.; Cho, D. H.; Chung, C.-M. Synlett 2001, 1923.

6. Bowman, W. R.; Krintel, S. L.; Schilling, M. B. Org. Biomol. Chem. 2004, 2, 585.

7. Vaillard, S. E.; Postigo, A.; Rossi, R. A. J. Org. Chem. 2004, 69, 2037.

8. Miura, K.; Ootsuka, K.; Hosomi, A. Synlett 2005, 3151.

9. Ishibashi, H.; Sato, T.; Ikeda, M. Synthesis 2002, 695.

10. Ishibashi, H.; Nakamura, N.; Sato, T.; Takeuchi, M.; Ikeda, M. Tetrahedron Lett. 1991, 32, 1725.

11. Sato, T.; Ishida, S.; Ishibashi, H.; Ikeda, M. J. Chem Soc., Perkin Trans. 1 1991, 353.

12. Jones, K.; Thompson, M.; Wright, C. J. Chem. Soc., Chem. Commun. 1986, 115.

13. Wehrli, R.; Heimgartner, H.; Schmid, H.; Hansen, H.-J. Helv. Chim. Acta 1977, 60, 2034.

14. Sato, T.; Ito, T.; Ishibashi, H.; Ikeda, M. Chem. Pharm. Bull. 1990, $38,3331$.

15. Ishibashi, H.; Kameoka, C.; Iriyama, H.; Kodama, K.; Sato, T.; Ikeda, M. J. Org. Chem. 1995, $60,1276$. 